DOI: $10.1007 / s 00350-009-2412-z$

\section{Datenschutz in der Arztpraxis. Ein Leitfaden für den Umgang mit Patientendaten.}

Von Andreas Höpken und Helmut Neumann. Verlage C.F. Müller, Heidelberg u.a., und MedizinRecht.de, Frankfurt a.M., 2. Aufl. 2008, 96 S., Softcover, $€ 29,80$

Der Leitfaden (die Lizenzausgabe eines zunächst im DatakontextVerlag erschienenen Werkes) zielt auf eine praxisorientierte Hilfestellung für die niedergelassenen Ärzte und ihr Praxispersonal. Er beginnt mit einem hilfreichen kurzen Überblick über Zweck und Ziel des Datenschutzes, Historische Grundlagen, Entwicklung der Gesetzgebung, zentrale Begriffe des Datenschutzrechts und einschlägige Rechtsvorschriften. Eingehend wird dann zunächst das Thema "Schweigepflicht/Patientengeheimnis“ behandelt (S. 19ff., wobei die Auskunftspflicht des Arztes gegenüber dem Patienten eigentlich nicht in diesen Abschnitt gehört und ein eigener Abschnitt zu den Patientenrechten - Recht auf Auskunft, Einsicht, Berichtigung, Sperrung, Löschung - angemessen wäre). Für eine Vielzahl in der täglichen Praxis relevanter Situationen werden detailliert und verständlich Risiken für den Datenschutz und Voraussetzungen einer befugten Offenbarung personenbezogener Patientendaten dargelegt und damit eine grundlegende Orientierung für datenschutzgerechtes Verhalten ermöglicht. Vor dem Hintergrund unterschiedlicher landesrechtlicher Regelungen (z. B. Landeskrebsregistergesetze) oder komplexer rechtlicher Regelungen (z. B. Sozialgesetzbuch V) führt die Kürze der Darstellung allerdings teilweise zu Vereinfachungen und Unschärfen (z.B. S. 22 „Eine Offenbarung an den MDK ist in der Regel zulässig"). Das zunehmend wichtiger werdende Thema „Integrierte Versorgung“ wird zu kurz aufgegriffen (S. $25, \ldots$ im Zweifelsfall müssen die Patienten um Einwilligung gebeten werden“"): die einschlägigen detaillierten Regelungen der $\$ S 140$ ff. SGB V zur gesetzlich vorgeschriebenen gemeinsamen Behandlungsdokumentation der Leistungserbringer bei der Integrierten Versorgung und $\mathrm{zu}$ den Rechten der Patienten werden nicht dargestellt. Andere ebenfalls zunehmend wichtiger werdende Kooperationsformen der Leistungserbringer wie z.B. das Hausarztmodell, Ärztenetze und einrichtungsübergreifende elektronische Fallakten werden gar nicht thematisiert, obwohl auch und gerade im Hinblick auf diese neuen Kooperationsformen datenschutzgerechte Verfahrensweisen (sowohl in rechtlicher wie auch in technischer Hinsicht) in der Arztpraxis klärungsbedürftig sind. Ungeachtet dessen ist jedoch gerade dieser Abschnitt für die Förderung eines Datenschutzbewußtseins und eine generelle erste Orientierung sehr hilfreich; zur Ergänzung wichtig ist dabei auch der Hinweis, dass in Zweifelsfällen die Kassenärztliche Vereinigung, die Ärztekammer oder der Datenschutzbeauftragte konsultiert werden können. Leider wird bezüglich der zuständigen Datenschutzinstanz weder hier noch im Anhang klar dargestellt, dass für die niedergelassenen Ärzte die Aufsichtsbehörden für den nichtöffentlichen Bereich zuständig sind (www.bfdi.bund.de/nn_531524/ DE/AnschriftenUndLinks/AufsBehoerdFuerDenNichtOeffBereich/AnschriffenAufsichtsbehoerdenFue).

Dr. iur. Rita Wellbrock,

Der Hessische Datenschutzbeauftragte, Referatsleiterin Gesundheitswesen, Wissenschaft und Forschung, Betreuungsrecht, Wiesbaden, Deutschland
Angaben zu den Normen, in denen die jeweils erwähnten datenschutzrechtlichen Anforderungen festgelegt sind, sowie z.B. der Fundstellen für die erwähnten Entscheidungen des BVerfG könnte ein an weiteren Details interessierter Leser vermissen; der kursorische Überblick am Anfang über gesetzliche Grundlagen kann dies nicht ersetzen.

Die Strukturierung der im Folgenden angesprochenen Themenbereiche unter den Stichwörtern „Praxis“, „Lösungsansätze“ und „Die Praxis-EDV“ erscheint etwas verwirrend, es sind jedoch auch in diesen Abschnitten wieder vielfältige in der Alltagspraxis relevante Situationen, Risiken und Datenschutzanforderungen detailliert und verständlich angesprochen, z. B. datenschutzgerechtes Verhalten im Empfangs-, Warte- und Behandlungsbereich, bei der Benutzung von Telefon, Telefax und e-mail und bei der Entsorgung/Löschung von Daten. Detailliert werden auch die Anforderungen an eine rechtswirksame Schweigepflichtentbindung dargestellt (S. 42). Die Frage, inwieweit Ärzte in einer größeren Praxis wechselseitig die Daten ihrer Patienten zur Kenntnis nehmen dürfen, wird leider nicht thematisiert. Die Kürze der Darstellung führt ferner auch in diesem Teil dazu, dass die neuen Kooperationsformen (s. o.) nicht berücksichtigt werden.

Die kurzen Anmerkungen zur Einführung der elektronischen Gesundheitskarte (S. $84 \mathrm{ff}$.) sind weniger anschaulich als die übrigen Teile des Leitfadens und auch nicht fehlerfrei. So besteht z.B. das für das Datenschutzkonzept der elektronischen Gesundheitskarte zentrale Zwei-Schlüssel-System nicht aus dem PIN-Code des Patienten und einem elektronischen Heilberufsausweis, der Zugriff auf die freiwilligen medizinischen Daten ist vielmehr nur mittels zweier Karten - der elektronischen Gesundheitskarte des Versicherten und des elektronischen Heilberufsausweises des Arztes (ggf. zusätzlich PIN-Codes) - möglich. Die Autoren sehen die Gefahr, dass sich die Patienten, die ja keinen direkten Einfluss auf die technischen Komponenten und deren Anwendung haben, als reine Objekte der Datenverarbeitung degradiert fühlen könnten. Als Gegenmaßnahme wird eine umfassende Aufklärung der Patienten über die Chancen und Risiken der elektronischen Gesundheitskarte angesehen. Dies ist sicherlich zutreffend, über diese Aufklärung hinaus wird es jedoch insbesondere auf die noch nicht abschließend entschiedene konkrete Ausgestaltung der Entscheidungsrechte der Patienten (namentlich bei der elektronischen Patientenakte) und die Sicherstellung von Transparenz der Verarbeitungsvorgänge für die Patienten (z.B. durch für den Patienten gut nachvollziehbare Protokolle) - auch in der Arztpraxis - ankommen.

Insgesamt ermöglicht der Leitfaden Ärzten und Praxispersonal einen Einstieg in das Datenschutzthema auf anschauliche, verständliche und lösungsorientierte Weise. Kenntnisreiche Detaildarstellung wird durch Wiederholungsfragen, konkrete Vorschläge für Formulierungen und für Datensicherheitsmaßnahmen sowie einen Selbst-Check sinnvoll ergänzt. Angesichts der Kürze der Darstellung sind Hinweise auf weiterführende links und Literatur von besonderer Bedeutung. Die Zusammenstellung der links und der weiterführenden Literatur erscheint jedoch recht zufällig und lückenhaft. Aufgenommen werden sollte z.B. in jedem Fall bei den links das virtuelle Datenschutzbüro (www.datenschutz.de), das eine Fülle von Materialien zum Thema „Datenschutz im Gesundheitsbereich“ enthält, die (2008 aktualisierten) „Empfehlungen zur ärztlichen Schweigepflicht, Datenschutz und Datenverarbeitung“ der Bundesärztekammer und der Kassenärztlichen Bundesvereinigung (www.bundesaerztekammer.de) und das Grundschutzhandbuch des BSI (www.bsi.bund.de/gshb/). 